\title{
INFLUENCE OF OXYGEN IMPURITIES ON ELECTRICAL PROPERTIES OF FULLERENE $\mathrm{C}_{60}$
}

\author{
T. Rabenau, S. Roth and R.K. Kremer \\ Max-Planck-Institut $f$. Festkörperforschung \\ Heisenbergstr. 1, D-70569 Stuttgart, Germany
}

\begin{abstract}
We present high temperature dc, ac and contactless microwave conductivity results on solid-state $\mathrm{C}_{60}$ (films and crystals) from room temperature up to $850 \mathrm{~K}$. Heating pristine samples, which were exposed to the ambient atmosphere, under dynamic vacuum at first leads to a reduction of the electrical resistance and finally, above $\approx 700 \mathrm{~K}$, to an increase in the resistance. The decrease is ascribed to oxygen desorption and the increase to the chemical reactivity of residual chemisorbed oxygen with the $\mathrm{C}_{60}$ host molecules, respectively. Samples, annealed above $800 \mathrm{~K}$, display a reversible temperature dependence of the resistance. The high temperature regime of their resistance exhibits an activated behaviour with an universal activation energy of $2 E_{\mathrm{a}}=1.85 \pm 0.04 \mathrm{eV}$ for crystals and films, which is identical to the HOMO-LUMO splitting of the $\mathrm{C}_{60}$-molecules.
\end{abstract}

PACS numbers: 72.20.-i, 72.20.Jv, 72.80.Le

\section{Introduction}

Much research has been devoted to the studies of the interaction of oxygen with $\mathrm{C}_{60}$ fullerene [1-11]. Molecular oxygen is reported to be absorbed but not to react with the $\mathrm{C}_{60}$-molecules at room temperature (e.g. $\left.[6,7]\right)$. However, at temperatures above $470 \mathrm{~K}$ an oxidation of $\mathrm{C}_{60}$-molecules has been found [8].

In this paper we focus our interest on the effects of oxygen impurities on the electrical properties of $\mathrm{C}_{60}$-samples. Performing the resistance measurements under dynamical vacuum conditions, a decrease in the resistance is observed. It is ascribed to desorption of absorbed $\mathrm{O}_{2}$. The chemical reaction of residual oxygen dominating at temperatures above $\approx 700 \mathrm{~K}$ leads to an increase in the resistance. Thermal reversibility of the resistance of samples which were heated to temperatures above $800 \mathrm{~K}$ provides complete desorption and/or reaction of oxygen with $\mathrm{C}_{60}$-molecules. 


\section{Experimental}

Preparation of the crystals has been discussed previously [12]. Films were sublimed in a simple evaporation chamber using as substrate a thin sapphire plate with gold contacts [6]. All samples were exposed to ambient atmosphere (the crystals for several days) prior to measurement.

The $\mathrm{dc}$ and ac conductivities were determined in a furnace using a HP4274 LCR-meter $(100 \mathrm{~Hz}-100 \mathrm{kHz})$ or using a Keithley 617 programmable electrometer (two point method, test current $1 \mathrm{nA}$ ). The measurements were done during slow $(\approx 3 \mathrm{~K} / \mathrm{min}$ ) heating and cooling cycles. The microwave $(10 \mathrm{GHz})$ conductivities were obtained on $\mathrm{C}_{60}$-powders and on a set of small single crystals by means of the contactless microwave cavity perturbation method as described in detail elsewhere [13]. The furnace and the microwave sample capillary were continuously evacuated ( $p<10^{-2}$ mbar) during the measurements.

\section{Results and discussion}

Figure 1 displays the characteristic behaviour of the resistances of crystalline and film $\mathrm{C}_{60}$-samples. Starting a measurement with pristine (oxygenated) samples two competing effects characterize the $\mathrm{dc}$, ac and also microwave conductivities. At room temperature where no chemical reaction of oxygen with $\mathrm{C}_{60}$-molecules was detected [6, 7], there is the influence of absorbed molecular oxygen only. Lowering the amount of absorbed oxygen by pumping and/or heating reduces the resistance of the sample. This is in best agreement with measurements of electrical conductivities on $\mathrm{C}_{60}$-crystals [10] and $\mathrm{C}_{60}$-films [11], and of photoconductivity on $\mathrm{C}_{60}$-films [6] where absorbed oxygen was identified to act as effective recombination center for charge carriers. The result suggests that the conductivity is limited by the level of oxygen in the sample and can easily be improved by its removal. This effect is reversible (subsequent exposure of the sample to air causes an increase in resistance). Above $470 \mathrm{~K}$, the onset of a chemical reaction of oxygen with $\mathrm{C}_{60}$-molecules was reported [8]. This irreversible reaction is accompanied by an increase in the resistance. This effect can be clearly seen from the heating curves of pristine samples (Fig. 1). There, the minimum of resistance denotes the regime, where the effect of the chemical reaction on the conductivity begins to dominate. For films the minimum is located at $\approx 650 \mathrm{~K}$. This is somewhat different from bulk crystalline material where the minimum is shifted to about $740 \mathrm{~K}$. This shift may be due to the different morphology of the samples resulting in a different surface/volume ratio and different desorption time constants.

Above $800 \mathrm{~K}$ the increase in the resistance stops indicating that all oxygen either was desorbed or reacted with $\mathrm{C}_{60}$-molecules. The remaining resistance exhibits a thermally activated behaviour in the high temperature regime. Within the experimental error subsequent heating and cooling cycles do not alter the resistance any more. This also suggests that the sample does not change during the measurement, specially there is no significant sublimation of $\mathrm{C}_{60}$-material at higher temperatures. It is noticeable that the activation energy with $E_{\mathrm{a}} \approx 0.92 \mathrm{eV}$ is identical for all $\mathrm{C}_{60}$-samples suggesting that the residual conductivity is governed 


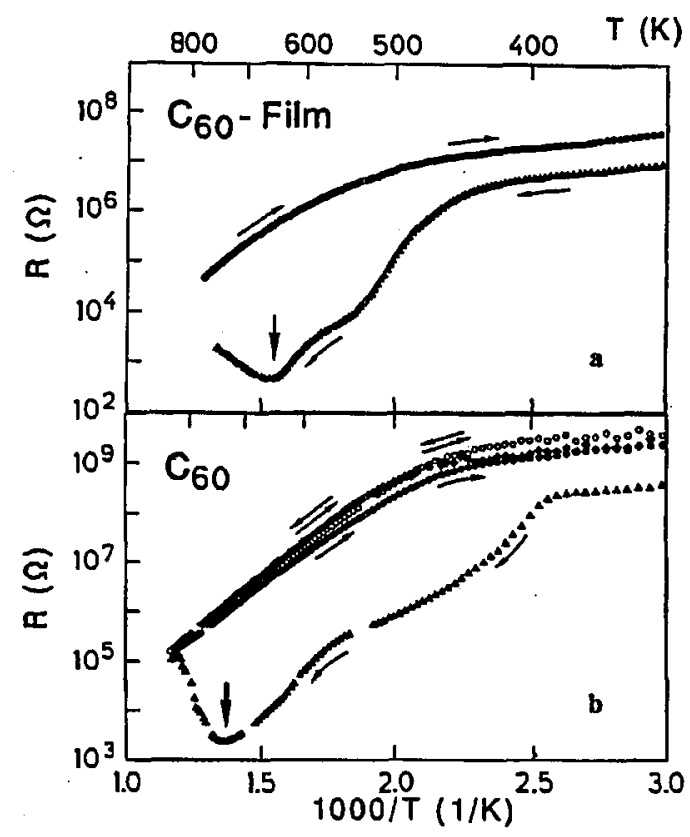

Fig. 1. (a) The ac resistance, $R$, at $4 \mathrm{kHz}$ of a sublimed $\mathrm{C}_{60}$-film versus reciprocal temperature. Lower curve (full triangles) is the initial heating cycle, upper curve ( $\bullet$ ) marks the (reversible) cooling cycle. (b) The ac resistance at $200 \mathrm{~Hz}$ of a $\mathrm{C}_{60}$-crystal. Lowest curve $(\Delta)$ is the initial heating cycle. The upper curves are the subsequent heating $(\bullet, 0)$ and cooling (full diamonds) cycles. The arrows indicate the direction of temperature change.

by intrinsic features of the intact $\mathrm{C}_{60}$-molecules, essentially the HOMO-LUMO (highest occupied molecular orbitals-lowest unoccupied molecular orbitals) splitting of the $\mathrm{C}_{60}$-molecules. Evaluating all our measurements [14] we obtain a gap of $\Delta E=2 E_{\mathrm{a}}=1.85 \pm 0.04 \mathrm{eV}$. This value for the HOMO-LUMO gap of the $\mathrm{C}_{60}$-molecules has been well established [15-17].

Although at low temperatures the resistance is only weakly temperature dependent, a strong frequency dependence is observed. This behaviour is attributed to extrinsic properties, which are sample dependent.

\section{Conclusions}

We investigated the influence of absorbed molecular oxygen impurities on the electrical conductivity of $\mathrm{C}_{60}$-samples. We performed $\mathrm{dc}$ and ac measurements on films and crystals as well as microwave absorption on crystallites and powder in the temperature range from $300 \mathrm{~K}$ to $850 \mathrm{~K}$. Heating pristine (oxygenated) samples in a dynamical vacuum causes a (reversible) loss of absorbed oxygen resulting in a reduction of the resistance. We observe that absorbed oxygen acts as a trap 
for charge carriers in $\mathrm{C}_{60}$-samples limiting their conductance. At about $700 \mathrm{~K}$, the (irreversible) chemical reaction of residual oxygen with $\mathrm{C}_{60}$-molecules becomes the dominant influence on the conductance causing a drastic decrease. The stop of this decrease indicates that all oxygen has desorbed from the sample or has reacted. The residual conductance exhibits the same thermally activated behaviour for all samples (crystals and films). Ascribing this effect to intrinsic features of the intact $\mathrm{C}_{60}$-molecules we determine the HOMO-LUMO splitting of $\mathrm{C}_{60}$-molecules to $\Delta E=1.85 \pm 0.04 \mathrm{eV}$. At lower temperatures, the conductance in each sample is governed by extrinsic effects.

\section{Acknowledgment}

We thank gratefully Lidia Akselrod for valuable discussions and Holger Diem for experimental help.

\section{References}

[1] H. Werner, D. Bublak, U.Göbel, B. Henschke, W. Bensch, R. Schlögl, Angew. Chem. 104, 909 (1992).

[2] S.J. Duclos, R.C. Haddon, S.H. Glarum, A.F. Hebard, K.B. Lyons, Solid State Commun. 80, 481 (1991).

[3] T. Pichler, M. Matus, J. Kürti, H. Kuzmany, Phys. Rev. B 45, 13841 (1992).

[4] H.S. Chen, A.R. Kortan, R.C. Haddon, M.L. Kaplan, C.H. Chen, A.M. Mujsce, H. Chou, D.A. Fleming, Appl. Phys. Lett. 59, 2956 (1991).

[5] M.K. Nissen, S.M. Wilson, M.L.W. Thewald, Phys. Rev. Lett. 69, 2423 (1992).

[6] M. Kaiser, W.K. Maser, H.J. Byrne, A. Mittelbach, S. Roth, Solid State Commun. 87, 281 (1993).

[7] L. Akselrod, M.Sc. Thesis, University of Dublin, Dublin 1993, p. 45.

[8] A.M. Vassallo, L.S.K. Pang, P.A. Cole-Clarke, M.A. Wilson, J. Am. Chem. Soc. 113,7820 (1991).

[9] L. Akselrod, H.J. Byrne, T.E. Sutto, S. Roth, submitted to Chem. Phys. Lett.

[10] T. Arai, Y. Murakami, H. Suematsu, K. Kikuchi, Y. Achiba, I. Ikemoto, Solid State Commun. 84, 827 (1992).

[11] A. Zahab, L. Firlej, Solid State Commun. 87, 893 (1993).

[12] M. Haluska, H. Kuzmany, M. Vybornov, P. Rogl, P. Fejdi, Appl. Phys. A 56, 161 (1993).

[13] T. Rabenau, A. Simon, R.K. Kremer, E. Sohmen, Z. Phys. B 90, 69 (1993).

[14] R.K. Kremer, T. Rabenau, W.K. Maser, M. Kaiser, A. Simon, M. Haluska, H. Kuzmany, Appl. Phys. A 56, 211 (1993).

[15] R. Zamboni, M. Muccini, R. Danieli, C. Taliani, H. Mohn, W. Müller, H.U. ter Meer, in: Fullerenes and Photonics, Ed. Z.H. Kafafi, Procedings SPIE, Vol. 2284, 1994, p. 120.

[16] W. Guss, J. Feldmann, E.O. Göbel, C. Taliani, H. Mohn, P. Haeusslerand, H.U. ter Meer, Phys. Rev. Lett. 72, 2648 (1994).

[17] B. Koopmans, A. Anema, H.T. Jonkman, G.A. Sawatzky, F. van der Wounde, Phys. Rev. B 48, 2759 (1991). 